\title{
Graphical User Interface Approach for Quality Evaluation of Indian Rice
}

\author{
Niky K. Jain \\ Research Scholar RKU, Rajkot \\ Department of M Sc. (IT) \\ ISTAR, V.V.Nagar
}

\author{
Samrat O. Khanna \\ Dean School of Engineering \\ and Applied Science \\ Rai University
}

\author{
Chetna K. Shah \\ Department of Electronics and \\ Communication \\ GCET
}

\begin{abstract}
Modernization with automization incorporated makes a system more powerful. In the present world quality inspection of food products is a very important factor for evaluating the grade of food. In agricultural field, image processing is also used to evaluate the quality of rice. Major problem of rice industry for quality assessment is manual assessment done by human inspector. In this paper a method is presented to evaluate the quality of rice. Proposed method is an application of computer vision technique. Computer Vision provides an alternative for non-destructive and cost effective technique for Grading and Classification of rice using framework and neural network techniques. Some Geometrics features are useful for quality evaluation. In this paper proposed method is used to increase the accuracy of the rice quality detection by using such features with GUI (Graphical User Interface) and feed forward neural network. Artificial neural network detects the quality of rice by using features provided at the time of training and also the extracted features of rice and provides the result by comparing these features. It grades and classifies rice images based on obtained features.
\end{abstract}

\section{Keywords}

Feature extraction, GUI (Graphical user interface), Image processing, Quality analysis.

\section{INTRODUCTION}

Agriculture field has played an important role in economic development of India. Digital Development in agriculture sector is growing exponentially as compared to development in other sector. So, there is need to develop some new technique for agricultural sector. There are various rice varieties are available in India like Basmati, Ponia, Masoori, Parimal, Jirasar, Kamod etc. Still in India, the traditional inspection of rice is performed by human experts. It is not only time consuming but a laborious technique too. As it is perceived to be a possible solution to prevent human errors in the quality evaluation process. Machine vision system which is a promising technology in the quality control can replace the human operator[1]. After hours of working the operator may lose concentration which in turn will affect the evaluation process. Hence a Machine vision system is proved to be more efficient at the level of precision and rapidity. But, the natural diversity in appearance of various rice varieties makes classification by Machine vision a complex work to achieve. Many researches were carried out to classify grains. Characterization models were based on morphological features, colour features or textural features[2]. Other researchers([20-21]) have tried to combine these features for the sake of improving the efficiency of classification. Recently, wavelet technique was integrated in grains characterization([22-23)].

Grading of rice is necessary in evaluating agricultural produce, meeting quality standards and increasing market value. The features that can be extracted from an image of any rice are its Major Axis, Minor Axis, Eccentricity, Area, Convex Area, Perimeter and Extent[17]. These features help the user to classify the rice. In this Paper, an automatic framework is used to analyse the rice quality which is based on Major Axis, Minor Axis, Eccentricity, Area, Convex Area, Perimeter and Extent. Rice should be tested via nondestructive techniques because these are delicate materials. If the classification and grading is done through manual methods, the procedure will be too moderate and at times it will be mistake inclined. Human choose the rice on the premise of bare eye perception[7]. On the off chance that these quality measures are mapped into computerized framework by utilizing appropriate programming dialect then the work will be speedier and blunder free. Lately, PC machine vision and picture preparing methods have been discovered progressively helpful in the agrarian business, particularly for applications in quality review and shape arranging. The exploration work abridged in this paper concentrates on the issue confronted by Indian Rice industry and its financially savvy arrangement. In this paper we have talked about different rice assortment (Oryza Sativa L) seeds containing various size of rice seeds available.

In this paper at first user select the type of rice variety like Basmati, Ponia, Masoori, Parimal, Jirasar, Kamod etc. and systems automatically evaluate the sample using its geometrical properties. Second elaborates the quality factor. Proposed methodology being used is enumerated using physical properties exploited from the image of the rice sample. The last three sections exemplify the result and discussion part based section 3 along with the data mining technique so used in our research work. With the help of this paper we propose a Framework for classification of rice.

\section{PROBLEM DEFINITION}

Automisation using a graphical user friendly system is introduced through this research paper. As previously the age old technique of quality evaluation was through mere naked eye inspection of few experts. It was not only biased but also inaccurate way. As the various rice varieties like Basmati, Ponia, Masoori, Parimal, Jirasar, available in this region comprise of different geometrical properties. They are unique in themselves in such a way that they make a wide difference with each other too. The before said thing is justified as shown in the first image of this paper. The different variety of rice in aforesaid sample degrade the quality of rice. In the below figure blue encircle one is a regular seed while the red encircled one is a small seed and green one is long seed. 


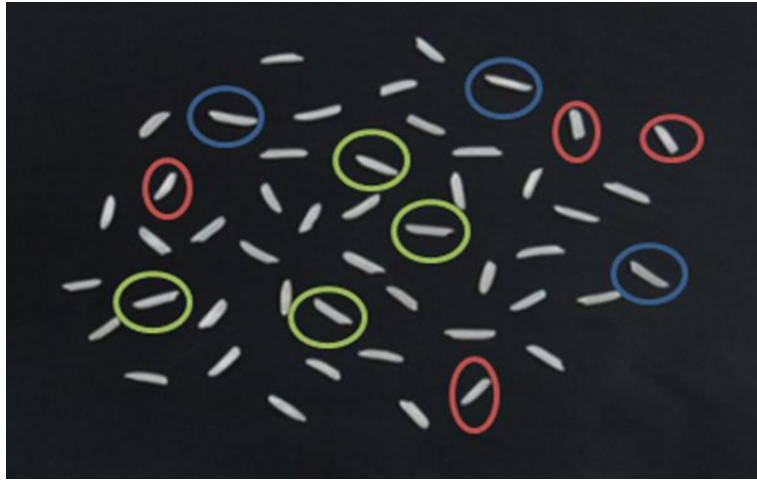

Fig. 1 Rice (Jirasar and Masoori) seeds with and without foreign elements

\section{SUGGESTED APPROACH}

This section elaborates the process of evaluation in a nonchemical environment. The proposed automated system is designed to overcome the problems of manual techniques. The system consists of several steps like feature extraction, sorting and grading. It is designed to combine seven processes as shown below in a flow table. We extract features for training the data and further we used this data for grading purpose. Proposed technique characterizes and perceives rice images based on obtained features values by using two-layer feed-forward network, with sigmoid covered up and yield neurons[15]. The image processing toolbox supports feed forward networks. The flow chart of sorting and grading process is given in the following Table 1 .

\section{Table 1 Suggested Approach}

\begin{tabular}{|l|l|}
\hline Sr. No. & Steps \\
\hline $\mathbf{1}$ & Sample of rice seeds \\
\hline 2 & $\begin{array}{l}\text { Image Acquisition } \\
\text { Convert over the RGB picture to dim picture. }\end{array}$ \\
\hline 3 & \begin{tabular}{l} 
Apply the edge recognition operation. \\
\hline 4
\end{tabular} \\
\hline 4 & Feature Extraction \\
\hline 5 & Sorting \\
\hline
\end{tabular}

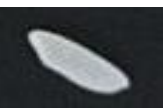

(i)

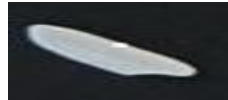

(ii)

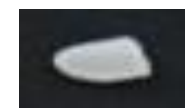

(iii)
Fig. 2 Sample comprising of various size of rice seeds

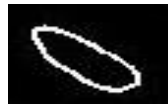

(i)

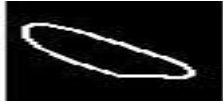

(ii)

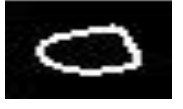

(iii)
Fig. 3 resultant rice seeds based on suggested approach

Actual type of rice seed appeared in figure 2(i), long sized appeared in figure 2(ii) and small sized appeared in figure 2(iii). Applying edge recognition operation on 2(i)-2(iii) rice seed and after we get pictures of figures 3(i), (ii) and (iii) individually. There are seven steps for the rice quality detection in proposed methodology. These steps are as following:

Step 1: To capture the image of given rice sample

Step 2: Load the image in GUI of Matlab
Step 3: After preliminary processing on the loaded image. Extract the geometrical features of rice sample for evaluation purpose

Step 4: Once the features are extracted then the soft computing technique of training the data set starts using feed forward neural network.

Step 5: Select the loaded rice sample for testing.

Step 6: Perform testing by using artificial neural network.

Step 7: Artificial neural network based output is achieved. It is a deciding parameter for classification of rice sample. As it explains in detail that the seeds so analyzed contained how much part of foreign elements and long seeds as well and the same is displayed in the count module of display section.

The first step is to acquire the image of rice. Image of the rice samples are captured by using a digital camera having 12 mega pixels quality with $8 \mathrm{X}$ optical zoom with black background mounted on the top of the box. The captured image is further stored in computer/laptop. The saved image is then loaded into the Matlab. Next comes the preprocessing of the loaded image in which image enhancement, noise removal are some of the key features of the process. In third step after edge detection the geometrical features of the rice samples are extracted. Geometrical features with the help of morphological operations are extracted. In fourth step neural network is used for training the data, after that in step fifth rice sample is selected for testing from database. In step sixth testing is performed by using ANN training module. Finally, in step seventh ANN based results are obtained.

\section{RESULTS ANALYSIS}

Table 2 represents parametric values of each rice seed. The values in the table displays the, Area (a), Major axis length (b), Minor axis length (c), Eccentricity (d), Convex area (e), Perimeter (f) and Extent (g). Similarly, other samples are found where each sample contains approximately 50 seeds are shown in table 3, 4 and 5. Table 2 represents analysis of Jirasar seeds, table 3 represents Masoori rice seeds and table 4 represents Parimal rice seed varieties respectively.

Table 2 Analysis for 15 seeds Available in One Sample

\begin{tabular}{|l|c|c|c|c|c|c|c|}
\hline S.No & $\mathbf{a}$ & $\mathbf{b}$ & $\mathbf{c}$ & $\mathbf{d}$ & $\mathbf{e}$ & $\mathbf{f}$ & $\mathbf{g}$ \\
\hline 1 & 155 & 36.45 & 17.17 & 0.88 & 333 & 74.08 & 0.25 \\
\hline 2 & 152 & 36.47 & 14.76 & 0.91 & 299 & 71.84 & 0.27 \\
\hline 3 & 149 & 35.09 & 13.89 & 0.91 & 277 & 70.28 & 0.38 \\
\hline 4 & 180 & 40.03 & 14.83 & 0.92 & 340 & 80.04 & 0.42 \\
\hline 5 & 150 & 32.41 & 14.62 & 0.89 & 274 & 67.45 & 0.41 \\
\hline 6 & 177 & 38.83 & 17.55 & 0.89 & 370 & 78.04 & 0.41 \\
\hline 7 & 178 & 35.76 & 16.31 & 0.88 & 333 & 74.04 & 0.44 \\
\hline 8 & 165 & 38.31 & 15.68 & 0.91 & 337 & 77.35 & 0.31 \\
\hline 9 & 173 & 38.98 & 16.75 & 0.90 & 361 & 78.91 & 0.26 \\
\hline 10 & 167 & 39.10 & 15.76 & 0.91 & 343 & 78.18 & 0.29 \\
\hline
\end{tabular}

Table 3 Analysis for 15 seeds Available in other Sample

\begin{tabular}{|c|c|c|c|c|c|c|c|}
\hline S.No & $\mathbf{a}$ & $\mathbf{b}$ & $\mathbf{c}$ & $\mathbf{d}$ & $\mathbf{e}$ & $\mathbf{f}$ & $\mathbf{g}$ \\
\hline 1 & 180 & 35.55 & 18.65 & 0.85 & 364 & 75.59 & 0.31 \\
\hline 2 & 179 & 37.32 & 18.76 & 0.86 & 381 & 77.69 & 0.37 \\
\hline 3 & 182 & 35.18 & 18.66 & 0.84 & 372 & 76.18 & 0.33 \\
\hline 4 & 169 & 38.09 & 17.61 & 0.88 & 361 & 76.66 & 0.27 \\
\hline 5 & 176 & 38.43 & 19.34 & 0.86 & 384 & 78.08 & 0.27 \\
\hline 6 & 182 & 39.42 & 20.08 & 0.86 & 419 & 82.42 & 0.27 \\
\hline 7 & 164 & 36.05 & 19.25 & 0.84 & 364 & 75.35 & 0.30 \\
\hline 8 & 162 & 37.34 & 18.72 & 0.86 & 367 & 76.18 & 0.29 \\
\hline 9 & 166 & 36.75 & 18.714 & 0.86 & 368 & 76.42 & 0.29 \\
\hline 10 & 162 & 34.76 & 15.78 & 0.89 & 308 & 70.38 & 0.46 \\
\hline
\end{tabular}


Table 4 Analysis for 15 seeds available in third Sample

\begin{tabular}{|c|c|c|c|c|c|c|r|}
\hline S.No & $\mathbf{a}$ & $\mathbf{b}$ & $\mathbf{c}$ & $\mathbf{d}$ & $\mathbf{e}$ & $\mathbf{f}$ & $\mathbf{g}$ \\
\hline 1 & 192 & 42.34 & 19.41 & 0.88 & 444 & 86.52 & 0.32 \\
\hline 2 & 191 & 41.75 & 19.06 & 0.88 & 431 & 84.28 & 0.36 \\
\hline 3 & 166 & 34.18 & 19.84 & 0.81 & 368 & 75.1 & 0.32 \\
\hline 4 & 196 & 40.81 & 20.38 & 0.86 & 453 & 85.94 & 0.32 \\
\hline 5 & 204 & 46.08 & 16.00 & 0.93 & 428 & 92.52 & 0.34 \\
\hline 6 & 208 & 45.49 & 18.14 & 0.91 & 455 & 91.11 & 0.34 \\
\hline 7 & 198 & 42.98 & 19.98 & 0.88 & 457 & 88.42 & 0.27 \\
\hline 8 & 188 & 40.32 & 17.89 & 0.89 & 395 & 81.49 & 0.27 \\
\hline 9 & 171 & 36.36 & 19.92 & 0.83 & 382 & 75.59 & 0.29 \\
\hline 10 & 170 & 37.21 & 18.59 & 0.86 & 373 & 76.62 & 0.37 \\
\hline
\end{tabular}

For classification of the three categories we compute thresholds values using the histogram. Table 5,6 and 7 mentions all the seven parameters and their computed threshold using histogram for area, major axis length, minor axis length, eccentricity, convex area, perimeter and extent of Jirasar, Masoori and Parimal rice seeds.

Table 5 Computed Threshold Values (Jirasar Rice)

\begin{tabular}{|c|c|c|c|}
\hline Features & Small seed & Normal seed & Long seed \\
\hline a & $110-140$ & $140-180$ & $180-200$ \\
\hline b & $25-31$ & $31-39$ & $39-45$ \\
\hline c & $10-14$ & $14-17$ & $17-25$ \\
\hline d & $0.65-0.84$ & $0.84-0.93$ & $0.93-1.00$ \\
\hline e & $200-270$ & $270-390$ & $390-410$ \\
\hline f & $50-66$ & $66-82$ & $82-90$ \\
\hline $\mathbf{g}$ & $0.2-0.25$ & $0.25-0.45$ & $0.45-0.50$ \\
\hline
\end{tabular}

Table 6 Computed Threshold Values (Masoori Rice)

\begin{tabular}{|c|c|c|c|}
\hline Features & Small seed & Normal seed & Long seed \\
\hline a & $125-150$ & $150-210$ & $210-235$ \\
\hline b & $25-32$ & $33-42$ & $42-47$ \\
\hline c & $14-17$ & $17-21$ & $21-24$ \\
\hline d & $0.65-0.77$ & $0.77-0.88$ & $0.88-0.92$ \\
\hline e & $280-320$ & $320-420$ & $420-460$ \\
\hline $\mathbf{f}$ & $50-68$ & $68-82$ & $82-90$ \\
\hline $\mathbf{g}$ & $0.2-0.26$ & $0.26-0.4$ & $0.4-0.50$ \\
\hline
\end{tabular}

Table 7 Computed Threshold Values (Parimal Rice)

\begin{tabular}{|c|c|c|c|}
\hline Features & Small seed & Normal seed & Long seed \\
\hline a & $120-155$ & $155-205$ & $205-250$ \\
\hline b & $25-32$ & $32-45$ & $45-51$ \\
\hline c & $12-15$ & $15-20$ & $20-25$ \\
\hline d & $0.75-0.84$ & $0.84-0.92$ & $0.92-1$ \\
\hline e & $300-350$ & $350-510$ & $510-550$ \\
\hline f & $65-75$ & $75-95$ & $95-105$ \\
\hline $\mathbf{g}$ & $0.2-0.24$ & $0.24-0.38$ & $0.38-0.4$ \\
\hline
\end{tabular}

Based on the above values of classification using histogram we achieved three categories of rice seeds in any given sample. It comprises of small, normal and large. So the quality of rice seeds get affected if concentration of any one either large seeds or small seeds are more in any random sample so taken. Hence to quantify the quality of any random rice sample the features so extracted are classified as discussed in next section.

\section{CLASSIFICATION}

\subsection{Graphical User Interface}

With MA TLAB we can make our own particular Graphical User Interface, or GUI, which comprises of a Figure window containing menus, catches, content, representation, and so on. That a client can control intelligently with the mouse and console. There are two fundamental strides in making a GUI: One is planning its design, and the other is composing call back works that play out the craved operations when the client chooses distinctive elements.

A graphical user interface (GUI) is a human-computer interface (i.e., a path for people to interact with computers) that utilizes windows, icons and menus and which can be controlled by a mouse (and regularly to a constrained degree by a console too). A GUI uses a blend of advances and gadgets to give a stage that the client can interface with, for the assignments of get-together and creating data. The most well-known mix of such components in GUIs is the WIMP ("window, icon, menu, and directing gadget") worldview, particularly in computers. Planning the visual synthesis and transient conduct of GUI is a vital part of programming applications. It will probably improve the productivity and usability for the fundamental legitimate configuration of a put away program, an outline discipline known as ease of use. Strategies for client focused outline are utilized to guarantee that the visual dialect presented in the outline is all around custom fitted to the undertakings.

\subsection{GUI Frame Work:}

\section{Flow of GUI Frame Work:}

Step 1: Click Pop-up menu and select type of rice.

Step 2: Select Image from child window.

Step 3: Click Upload File Push button.

Step 4: Click Edge Detection Push button.

Step 5: Automatic feature Extraction from different push button.

Step 6: Classification of Feature Extraction Value.

Step 7: Results.

As discussed in the flow of the framework first is to open the respective GUI as shown in figure 4.

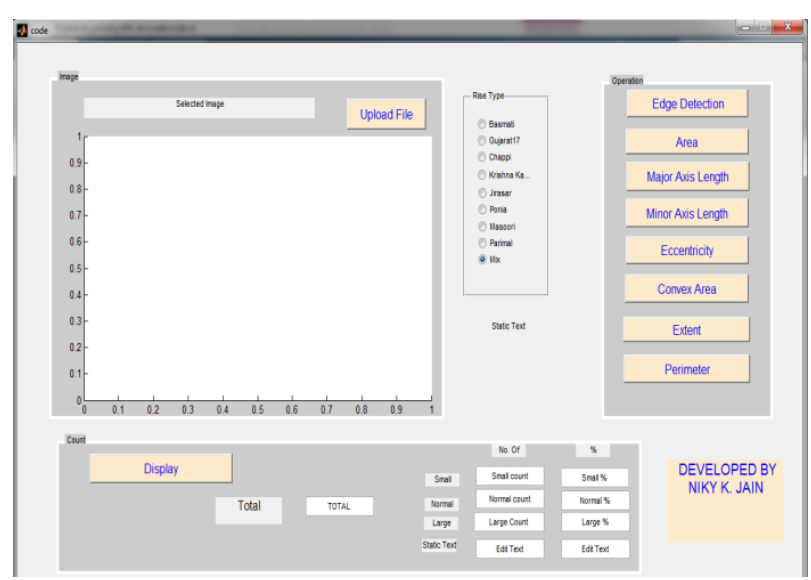

Fig. 4 GUI Framework

From the drop down menu one need to select the path of the folder where the images have been stored. Later that particular folder would be opened and one can select the given sample of seed image as shown in figure 5 . 
Fig. 5 Selection of a rice variety from the given folder

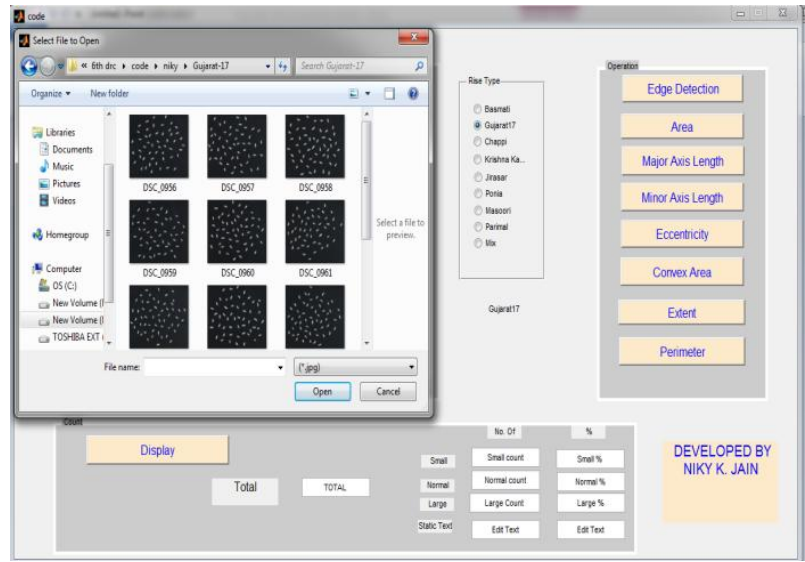

The image would then be uploaded on the given framework for further processing of information as shown in figure 6 .

Fig. 6 Uploading of a Rice sample

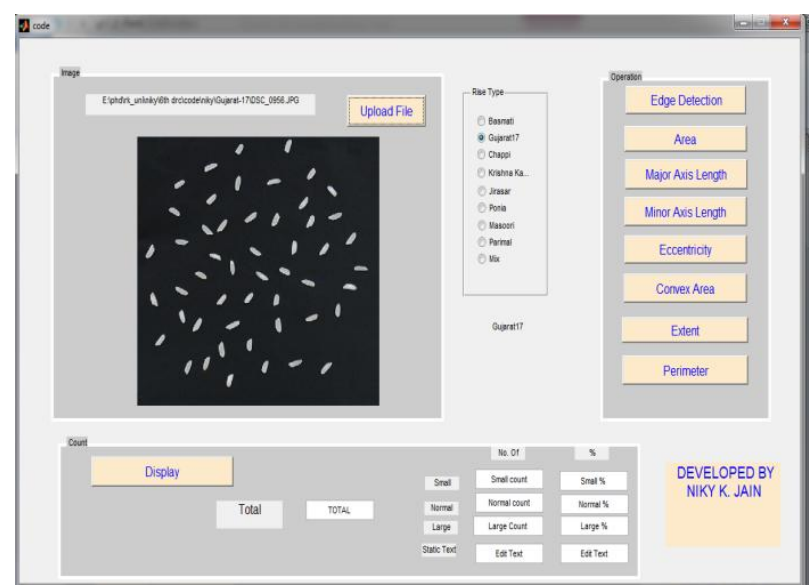

The image is preprocessed and edges of the image are detected as shown in figure 7 .

Fig. 7 Edge detected Rice sample

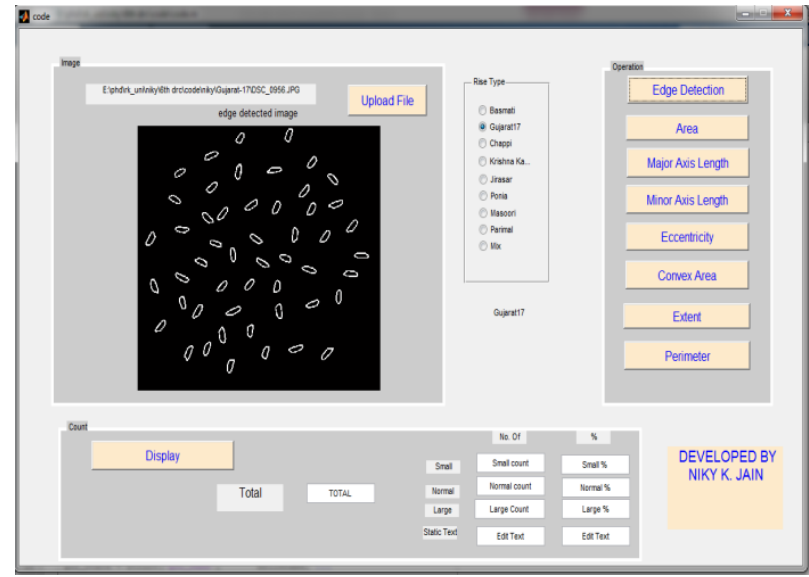

The edge detected rice sample is further processed and using morphological and geometrical operators the histogram of each sample is generated as shown in figure 8 to figure 16 . Each below shown figure represents the histogram of Area, Major Axis Length, Minor Axis Length, Eccentricity, convex Area, Extent and Perimeter respectively. Based on these histograms the features of rice seeds are extracted and calculated further.

Fig. 8 Histogram of Area of given Rice sample

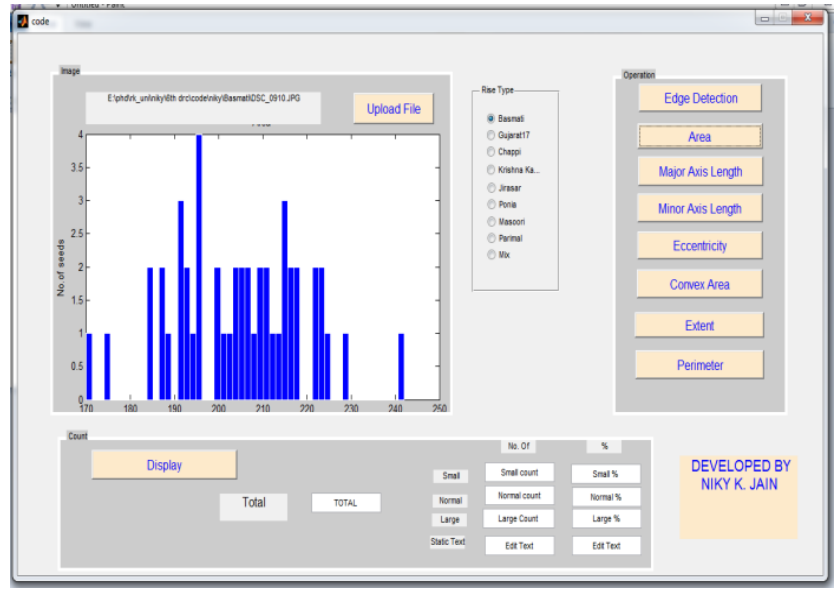

Fig. 9 Histogram of Major Axis Length of given Rice sample

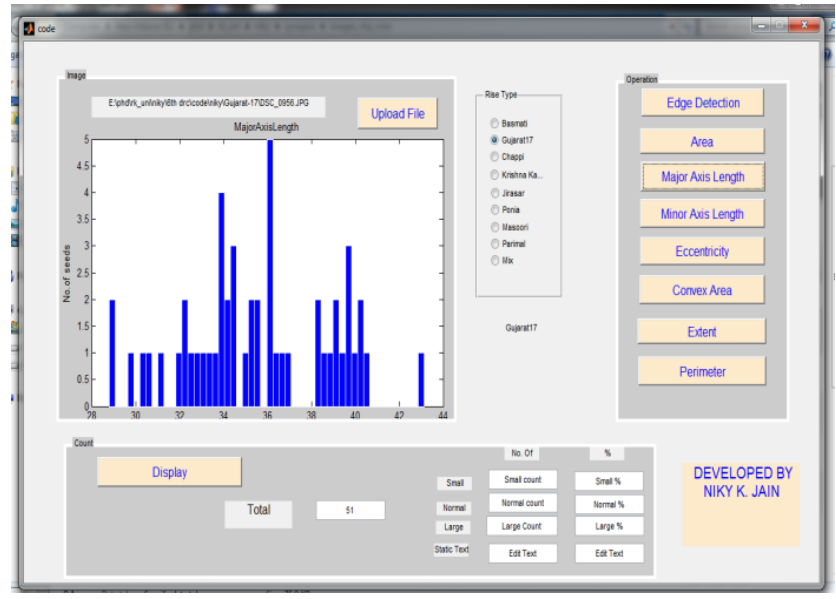

Fig. 10 Histogram of Minor Axis Length of given Rice sample

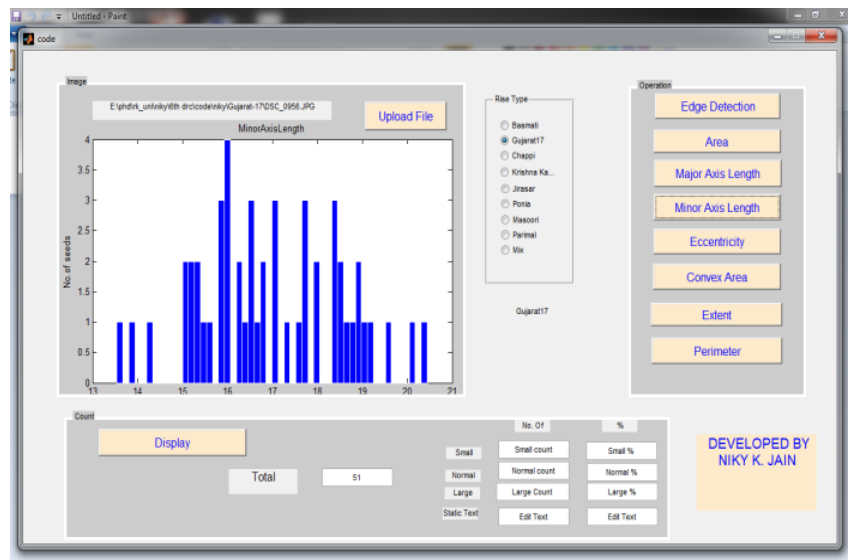


Fig. 11 Histogram of Eccentricity of given Rice sample

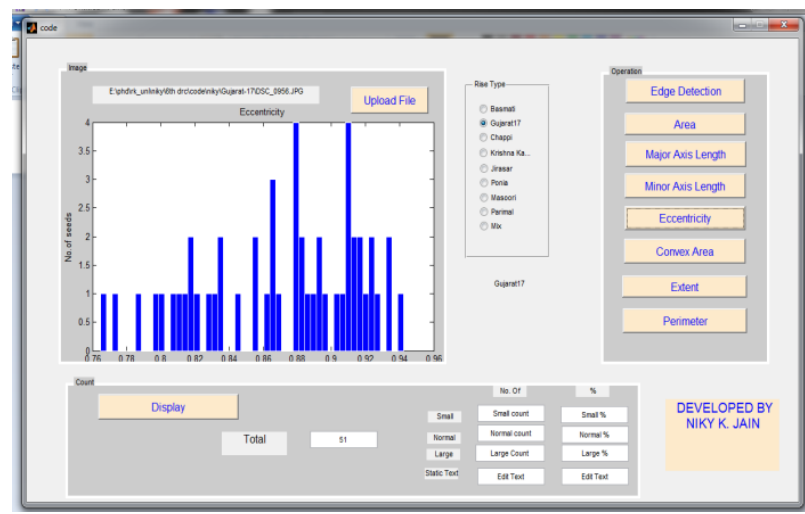

Fig. 12 Histogram of Convex Area of given Rice sample

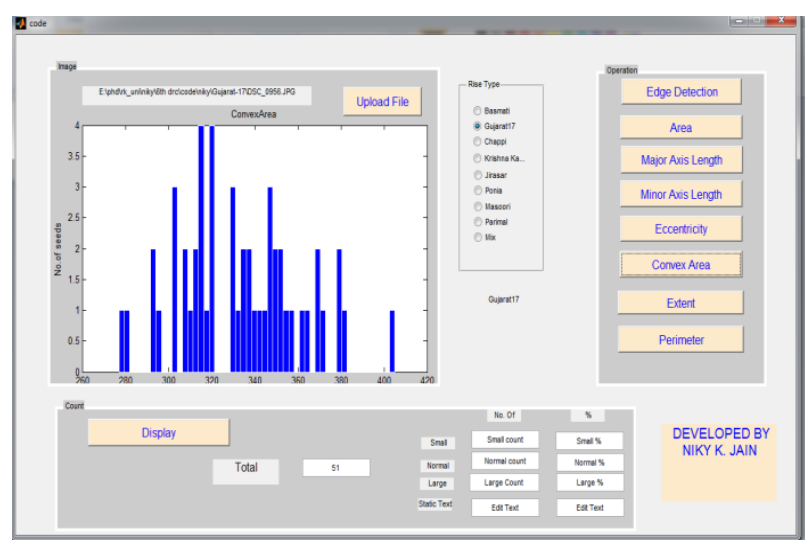

Fig. 13 Histogram of Extent of given Rice sample.

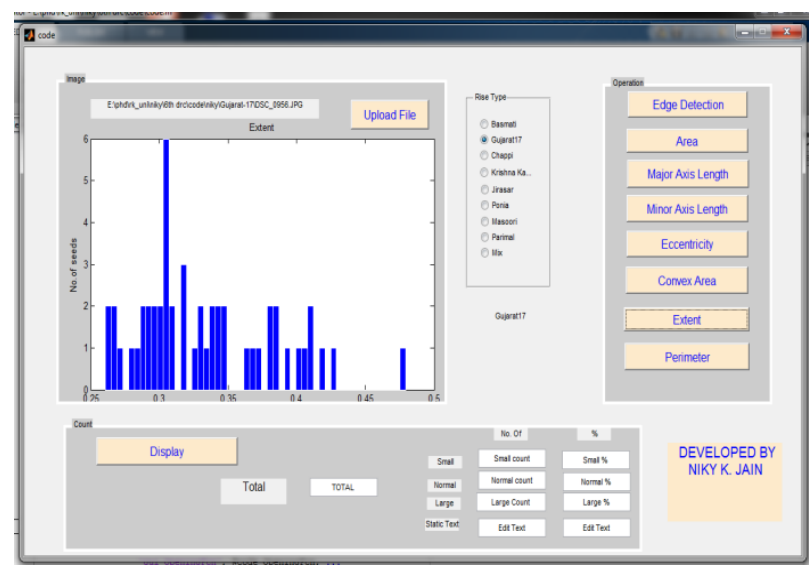

Fig. 14 Histogram of Perimeter of given Rice sample

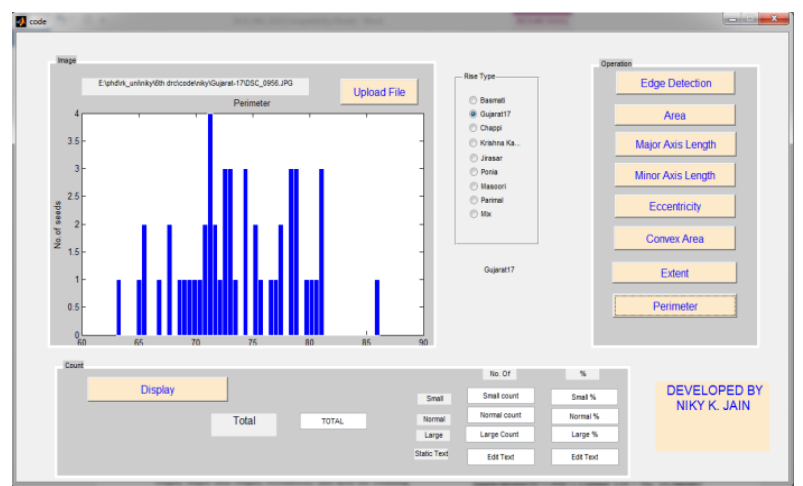

Based on the histograms so generated the calculation of small, normal and large seeds are done. It would help us calculate the quality of overall rice samples as shown in figure 14 .

Fig. 15 Display of large normal and small seeds of given Rice sample

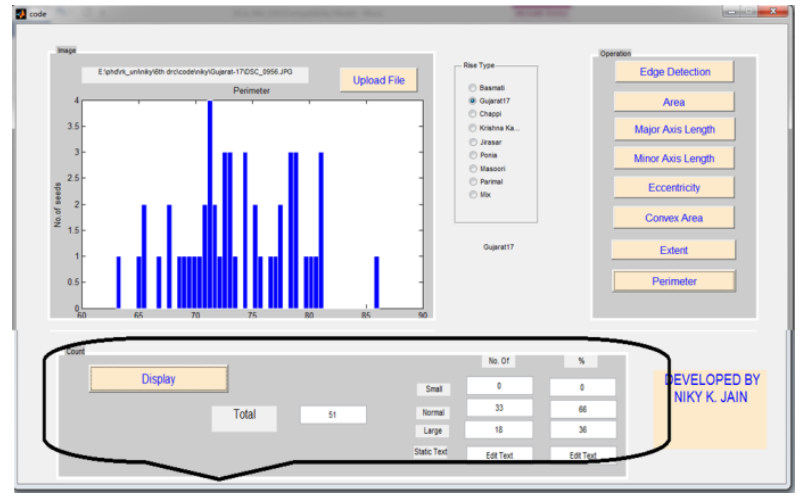

\section{CONCLUSION}

Quality quantification of Indian rice seeds using computer vision analysis and soft computing techniques have given an advancement in the agricultural sector of India. Here we were able to calculate geometrical features with the proposed approach. With the help of data mining approach using feed forward neural network provides an accuracy of $97 \%$ and was quiet effectively used in the rice mills of nearby areas.

\section{REFERENCES}

[1] Anami, Basavaraj S. and Savakar, Dayanand G. (2009) "Recognition and Classification of Food Grains, Fruits and Flowers Using Machine Vision,"International Journal of Food Engineering: Vol. 5: Issue. 4, Article 14.DOI: $10.2202 / 1556-3758.1673$

[2] Sagare, Sunil N. Kore, "Fruits Sorting and Grading Based on Color and Size", International Journal of Emerging Technologies in Computational and Applied Sciences (IJETCAS), IJETCAS 12-333.

[3] Ballard, D. A., \& Brown, C. M. " Computer vision", Englewood Cliffs, NJ, USA: Prentice-Hall ,1982.

[4] Niky K. Jain,Samrat O. Khanna,Chetna Maheshwari,," A Machine Vision Approach For Indian Basmati Rice Classification Using Geometric Features And CART" International Conference on signal and speech processing(ICSSP)-2014 (ELSEVIER digital library), Pages : 176-182,2014/8

[5] Chetna Maheshwari, Kavindra Jain, Chintan Modi, "Novel approach for Oryza sativa L.(Rice) based on Computer vision technology," PEPCCI, National Conference ,ISBN No.-978-93-81286-06-7,2012.

[6] Du CJ, Sun D-W, "Recent development in the applications of image processing techniques for food quality evaluation." Trends in Food Science and Technology, 15,230-249, 2004.

[7] Niky K. Jain,Samrat O. Khanna,Chetna Maheshwari "Development of a Classification System for Quality Evaluation of Oryza Sativa L.(Rice) Using Computer Vision", Communication Systems and Network Technologies (CSNT), 2014 Fourth International Conference on, Pages:1088-1092,2014/4/7, IEEE. 
[8] Gunasekaran Sundaram, Kexiang Ding, "Computer vision technology for food quality assurance,". Trends in Food Science and Technology, 7, 245-256, 1996.

[9] Jain AK, "Fundamentals of Digital Image Processing," Englewood Cliffs: Prentice-Hall 1989.

[10] Kavindra Jain, Chintan K. Modi, Kunal Pithadiya, "Non Destructive quality evaluation in spice industry with specific reference to Cuminum Cyminum L (Cumin) seeds," International Conference on Innovations \& Industrial Applications, Malaysia, (IEEE) 2009.

[11] M. Kurita and N. Kondo, "Agricultural product grading method by image processing (part 1) - effectiveness of direct lighting method", J.SHITA 18(1): 9-17,2006.

[12] Shen Castan, Sian Zhao,"A Comparitive study of Performance of Noisy roof edge detection", 5th International conference on Computer analysis of Images and Patterns, volu.179, pp 170-174

[13] Tadhg Brosnan, Da-Wen Sun, "Improving quality inspection of food products by computer vision-a review", Journal of Food Engineering 61, pp. 3-16, 2004.

[14] Xiaopei Hu, ParmeshwaraK.M, DavidV. "Development of Non Destructive Methods To Evaluate Oyster Quality By Electronic Nose Technology", Springer Science Business Media, LLC, 2008.

[15]Niky K. Jain,Samrat O. Khanna,Chetna Maheshwari,,"Feed Forward Neural Network Classification for INDIAN Krishna Kamod Rice"International Journal of Computer Applications(0975-8887),Volume 134 - No. 14,January 2016.

[16] Abutaleb AS, "Automatic thresholding of grey-level pictures using two-dimensional entropies." Pattern Recognition, 47(1), 22-32, 1989.

[17] Niky K. Jain,Samrat O. Khanna,Chetna Maheshwari,," A Machine Vision Approach For Indian Basmati Rice Classification Using Geometric Features And CART" International Conference on signal and speech processing(ICSSP)-2014 (ELSEVIER digital library), Pages : 176-182,2014/8

[18] Blasco J, Aleixos N, Molt E, "Computer vision system for automatic quality grading of fruit", Biosystems Engineering, 415-423, 2003.

[19] Du C-J, Sun D-W, "Learning techniques used in computer vision for food quality evaluation: a review", Journal of Food Engineering, 72(1), 39-55, 2006.

[20] Visen, N.S., Jayas, D.S., Paliwal, J., White, N.D.G.: Comparison of two neural network architectures for classification of singulated cereal grains. Canadian Biosystems Engineering 46, 3.7-3.14

[21] Douik, A., Abdellaoui, M.: Cereal varieties classification using wavelet techniques combined to multi-layer neural networks. In: Proc. 16th Mediterranean Conference on Control and Automation, Ajaccio, France, pp. 18221827 (2008)

[22] Chtioui, Y., Panigrahi, S., Backer, L.F.: Rough sets theory as a pattern classification tool for quality assessment of edible beans. Trans. of the ASAE 42(4), 1145-1152 (1999)

[23] Mallat, S.G.: A theory for multiresolution signal decomposition: the wavelet representation. IEEE Transactions on Pattern Analysis and Machine Intelligence 11(7), 674-693 (1989) 